\title{
SOME UNIVERSALLY PIERCED ARCS IN $E^{3}$
}

\author{
L. D. LOVELAND
}

ABSTRACT. A subset $X$ of $E^{3}$ is said to be universally pierced if each 2-sphere containing $X$ can be pierced by a tame arc at each point of $X$. We show that an arc $A$ is universally pierced provided $A$ has a shrinking point $p$ such that either $p$ lies in a tame arc in $A$ or $E^{3}-A$ has 1-ALG at $p$. Applying this result we show the existence of infinitely many wild universally pierced arcs.

Introduction. A subset $X$ of $E^{3}$ is called a pierced set if there exists a 2-sphere $S$ in $E^{3}$ containing $X$ such that $S$ can be pierced by a tame arc at each point of $X$. For example, a tame arc is universally pierced [5, Theorem 5] and consequently is a pierced set. The results in this paper support the following conjecture.

Conjecture. If $X$ is a pierced arc in $E^{3}$, then $X$ is universally pierced.

The truth of the conjecture would mean that the property of being pierced by a tame arc at a point of the arc $X$ is independent of the 2-sphere that contains $X$. In this note we give sufficient conditions, in terms of the following definitions, for an arc to be universally pierced.

$A$ point $p$ of a set $X$ is said to be a shrinking point of $X$ if, for every open set $U$ containing $X-\{p\}$, there is an open set $V$ containing $X-\{p\}$ such that each loop in $V-X$ shrinks to a point in $U-X$. For example, the Fox-Artin arc [4] $X$, which is wild at a single point $p$, has $p$ as a shrinking point but has no other shrinking point.

The complement of an arc $X$ in $E^{3}$ is said to have 1-ALG (abelian local fundamental group) at a point $p$ of $X$ if, for each open set $U$ containing $p$, there is an open set $V$ such that $p \in V \subset U$ and each loop in $V-X$ which bounds homologically ( $Z$ coefficients) in $U-X$ also bounds a singular disk in $U-X$. If $p$ is an endpoint of an arc $X$, then this simply says that $E^{3}-X$ is 1-LC at $p$. If the complement of an arc $A$ in $E^{3}$ has 1-ALG at each of its 1974.

Received by the editors December 19, 1973 and, in revised form, February 26,

AMS (MOS) subject clas sifications (1970). Primary 57A10, 55A30.

Key words and phrases. Piercing points, pierced sets, universally pierced, tame and wild arcs, surfaces in $E^{3}$, shrinking points, 1-ALG sets, cellular arcs. 
points, then $A$ is known to be tame [3, Theorem 3.16]. In the example mentioned above [4], the complement of the arc has 1-ALG at every point except at the shrinking point $p$.

The main theorems. The following lemma shows that to prove that a set $X$ is universally pierced it suffices to consider only 2-spheres that contain $X$ and that are locally tame modulo $X$. It follows from this lemma and [9] that Rosen's special arc [9] is universally pierced; however, this result also follows from Theorem 1 .

Lemma 1. If $X$ is an arc in $E^{3}$ such that every 2-sphere containing $X$ that is locally tame modulo $X$ can be pierced by a tame arc at each point of $X$, then $X$ is universally pierced.

Proof. Let $S$ be a 2-sphere containing $X$. (If there is no such sphere, then $X$ vacuously satisfies the definition of "universally pierced".) Using Bing's side approximation theorem relative to the open set $S-X$ (see [2, Theorem 4.6.5]) we find two 2-spheres $S_{1}$ and $S_{2}$, each locally tame modulo $X$ and lying (except for $X$ and the unions $H_{1}$ and $H_{2}$ of pairwise disjoint collections of disks in $S_{1}$ and $S_{2}$, respectively) in opposite complementary domains of $S$. Let $p$ be in $X$. By hypothesis, $S_{1}$ and $S_{2}$ can each be pierced by a tame arc at $p$. It follows [5, Theorem 6] that tame arcs $A_{1}$ and $A_{2}$ exist in $S_{1}-H_{1}$ and $S_{2}-H_{2}$, respectively, such that $p$ is an endpoint of both $A_{1}$ and $A_{2}$ and $A_{1} \cap X=A_{2} \cap X=\{p\}$. Thus $S$ is accessible by a tame arc from each of its complementary domains, and it follows from [7, Theorems 2 and 3] that $S$ can be pierced by a tame arc at $p$.

Theorem 1. If an arc $A$ in $E^{3}$ has a shrinking point $p$ and $E^{3}-A$ bas 1-ALG at $p$, then $A$ is universally pierced.

Remarks on Theorem 1. The condition " $E$ " $-A$ has $1-A L G$ at $p$ " cannot be removed since the Fox-Artin example [4] satisfies the other hypotheses and is clearly not universally pierced. However, if $A$ is required to be a pierced set with a shrinking point, then $A$ ought to be universally pierced. The arc described by Alford [1] satisfies these conditions but $E^{3}-A$ does not have 1-ALG at the shrinking endpoint; hence we have no proof that Alford's arc is universally pierced.

Proof of Theorem 1. By Lemma 1 it suffices to consider a 2-sphere $S$. containing $A$ where $S$ is locally tame modulo $A$. We first use the " $E^{3}-A$ has 1-ALG" hypothesis to show that $S$ can be pierced by a tame arc at $p$. For convenience we assume that $p$ is not an endpoint of $A$. 
Let $U^{\prime}$ be an open set containing $p$, and let $D$ be a disk in $S \cap U^{\prime}$ such that $p \in \operatorname{Int} D$ and $A \cap \operatorname{Bd} D$ is a two-point set. Let $U$ be an open set containing $p$ such that $U \cap S \subset$ Int $D$ and $U \subset U^{\prime}$, and let $C=S \cup$ Int $S$. We shall exhibit an open set $V$ containing $p$ such that each loop in $V \cap(C-\{p\})$ is homotopic to a constant in $U^{\prime} \cap(C-\{p\})$. The same argument will work for $C^{*}=S \cup$ Ext $S$, so it will then follow from McMillan's characterization of piercing points [7, Theorem 1] that $S$ can be pierced by a tame arc at $p$. Since $E^{3}-S$ is 1-ulc [10, Chapter X], there must be an open set $V^{\prime}$ such that $p \in V^{\prime} \subset U$ and each loop in $V^{\prime}-S$ bounds homologically in $U-S$. Now choose an open set $V$ such that $p \in V \subset V^{\prime}$ and each loop in $V-A$ that bounds homologically in $U-A$ also contracts in $U-A$.

Let $l$ be a loop in $V \cap(C-\{p\})$. Since $l$ can be moved with a homotopy (in $C-\{p\})$ into Int $C$, we may assume that $l$ lies in Int $C$. Since $l$ is a loop in $V^{\prime}-S$, it bounds homologically in $U-S$ and consequently in $U-A$. Then by the choice of $V, l$ contracts in $U-A$. Let $E$ be a singular disk in $U-A$ bounded by $l$ and notice that $E \cap S \subset$ Int $D-A$. From the construction of $D$ we see that $E \cap S$ lies in the union $D^{\prime}$ of the interiors of two disks in $D-A$. By Theorem 4.2.2 of [2] we may adjust $E$ to a new singular disk $E^{\prime}$ where $E^{\prime} \subset E \cup D^{\prime}, E^{\prime} \subset C$, and $l$ bounds $E^{\prime}$. Thus $l$ contracts in $U^{\prime} \cap$ $(C-\{p\})$, as we wished to show.

We must now show that $S$ can be pierced at every point of $A$. The fact that $p$ is a point where $S$ can be pierced by a tame arc allows us to find a tame arc $B$ in $S$ such that $B \cap A=\{p\}[5$, Theorem 6]. Let $q$ be the endpoint of $B$ different from $p$, and let $G$ be a disk in $S-A$ such that $G \cap B=\{q\}$. We shall construct a new 2-sphere $S^{\prime}$ by removing the interior of the tame $\operatorname{disk} G$ from $S$ and replacing it with a new disk $F$. Since $G$ is well away from $A$ it is clear that $S$ and $S^{\prime}$ share the same piercing properties at $A$. The $\operatorname{disk} F$ is constructed so that it is tame except at a point $x$ in Int $F$ where it is wild (see [2, Figure 3, p. 270]), Bd $F=\mathrm{Bd} G$, and $F \cap S \subset G$. Since $S^{\prime}=(S-G) \cup F$, it is clear that $S^{\prime}$ cannot be pierced by a tame arc at the isolated wild point $x$.

Let $T$ be the union of $A \cup B$ with an arc $R$ in $F$, where $R$ has $q$ and $x$ as its endpoints. Once we show that $T$ is cellular, it will follow from [8, Theorem 3] (or from [6, Theorem 1] if $F$ is placed in the appropriate component of $E^{3}-S$ ) that $T$ contains at most one nonpiercing point of $S^{\prime}$. The theorem then follows since $x$ is a known nonpiercing point of $S^{\prime}$.

To show that $T$ is cellular we let $U$ be an open set containing $T$, and we will indicate how to obtain a 3 -cell $M$ in $U$ with $T \subset$ Int $M$. Let $W$ be a 
tame ball centered at $p$, lying in $U$, whose boundary intersects $B$ at a single point; and let $V$ be an open set containing $A-\{p\}$ such that loops in $V-A$ shrink in $U-T$ and $V \cap(B \cup R)=\varnothing$. There is a finite collection $\left\{D_{1}\right.$, $\left.D_{2}, \cdots, D_{n}\right\}$ of disjoint disks on $\mathrm{Bd} W$ whose interiors cover $A \cap \mathrm{Bd} W$ such that each $D_{i}$ lies in $V$. Each Bd $D_{i}$ bounds a singular disk $E_{i}$ in $U-$ $T$, so (Bd $\left.W-\bigcup D_{i}\right) \cup\left(\bigcup E_{i}\right)$ is a singular 2-sphere $H$ which does not intersect $A$ and whose intersection with $B \cup R$ is the point of the set $B \cap \mathrm{Bd} W$. It is easy to construct a 3-cell $Y$ lying in $U$ with $B \cup R$ in its interior so that $H \cap B d Y$ is the union of three concentric simple closed curves $J_{1}, J_{2}$, and $J_{3}$ on $B d W$. (One begins with a round ball $Z$ centered at $x$ such that $R \cap \mathrm{Bd} Z$ consists of three points. Then three disks are found on $\mathrm{Bd} Z$ whose interiors contain $R \cap \mathrm{Bd} Z$. These disks are then pushed along $R \cup B$ and "over" $p$.) We add to $\mathrm{Bd} Y-\mathrm{Bd} W$ three singular disks in $H-T$ bounded by $J_{1}, J_{2}$, and $J_{3}$ to form a singular 2-sphere $K^{\prime}$ such that $K^{\prime} \subset U$, $K^{\prime} \cap T=\varnothing$, and $K^{\prime}$ separates $T$ from $E^{3}-U$. Now an application of the sphere theorem (see [2, Theorem 4.5.6]) yields a nonsingular polyhedral 2sphere $K$ with the same properties, and $K$ bounds a 3-cell $M$ containing $T$ in its interior. Since $K$ separates $T$ from $E^{3}-U$, we see that the 3-cell $M$ bounded by $K$ lies in $U$.

Corollary. Rosen's special arc [9] is universally pierced.

Theorem 2. If $A$ is an arc with a shrinking point $p$ and $p$ lies in a tame subarc of $A$, then $A$ is universally pierced.

Proof. If $p$ lies in the interior of a tame arc in $A$, then Theorem 2 follows directly from Theorem 1 . In any case, the proof of Theorem 1 gives the crucial steps. To see this, let $S$ be a 2-sphere containing $A$ and locally tame modulo $A$, and choose a tame arc $B$ such that $A \cap B=\{p\}$. Now a FoxArtin arc [4] $F$ is added just as in the proof of Theorem 1, and we see that $T=A \cup B \cup R$ is cellular the same way, even though it is not necessarily an arc. Again $T$ can contain at most the one nonpiercing point $x$ of the sphere $S^{\prime}$. Thus $S$ is pierced at each point of $A$ by a tame arc.

Applications. The results of the previous section can be used to produce infinitely many wild universally pierced arcs. We begin by considering the wild arc $A$ described by Alford [1] which has a shrinking endpoint $p$ (by its construction) and which lies on the boundary $S$ of a 3-cell in $E^{3}$. It is known that $p$ is a point where $S$ can be pierced by a tame arc; consequently there is a tame arc $B$ on $S$ such that $B \cap A=\{p\}$ [5]. Although we do not 
prove that Alford's arc $A$ is universally pierced, Theorem 2 shows that the arc $A_{t}=A \cup B$, formed by adding a tame arc to $A$, is universally pierced. This construction gives a hint for the construction of infinitely many such arcs.

Theorem 3. Alford's arc with a tame tail (described above) is universally pierced.

Theorem 4. There exist infinitely many inequivalent universally pierced arcs in $E^{3}$.

Proof. Let $S$ be a round sphere in $E^{3}$, and let $X$ be an arc in $S$ with endpoints $x$ and $y$. Let $\left\{D_{i}\right\}$ be a null sequence of disjoint disks on $S$ such that $\left\{D_{i}\right\}$ converges to $\{x\}$ and $D_{i} \cap X$ is a spanning arc $X_{i}$ of $D_{i}$. On each $D_{i}$ we construct an Alford arc $A_{i}$ such that $A_{i}$ is a spanning arc of a disk $E_{i}$ near $D_{i}$ where $\mathrm{Bd} E_{i}=\mathrm{Bd} D_{i}$ and $E_{i} \cap S \subset D_{i}$. The construction is done so that

$$
A=\left(X-\bigcup_{1}^{\infty} X_{i}\right) \cup\left(\bigcup_{1}^{\infty} A_{i}\right)
$$

is an arc from $x$ to $y$. For each $n$, let

$$
B_{n}=\left(X-\bigcup_{i=1}^{n} X_{i}\right) \cup\left(\bigcup_{i=1}^{n} A_{i}\right) \text {. }
$$

Each $A_{i}$ has a shrinking endpoint $p_{i}$, and we insist that $A_{i}$ be constructed so as to have $p_{i}$ the first point of $A_{i} \cap B_{n}(i \leq n)$ when $x$ is considered the first point of $B_{n}$. Then $p_{i}$ lies in a tame arc $T_{i}$ in $B_{n}$, and the arc $A_{i} \cup$ $T_{i}$ is universally pierced by Theorem 3 . Since $B_{n}$ is the union of universally pierced arcs, it is also universally pierced. Of course $B_{n}$ and $B_{m}$ $(n \neq m)$ are not equivalently embedded in $E^{3}$ since the wild set in $B_{n}$ has $n$ components while $B_{m}$ 's wild set has $m$ components.

Questions. We believe that all of the following questions have affirmative answers. Three of these questions are merely special cases of the conjecture stated earlier.

1. If an $\operatorname{arc} A$ is a pierced set and $A$ is cellular, then is $A$ universally pierced?

2. Is Alford's arc [1] universally pierced? This question is equivalent to one of Rosen's by Lemma 1.

3. If an $\operatorname{arc} A$ is a pierced set and $A$ has a shrinking point, then is $A$ universally pierced? 
4. Is every pierced nondegenerate continuum universally pierced?

\section{REFERENCES}

1. W. R. Alford, Some "nice" wild 2-spheres in $E^{3}$, Topology of 3-Manifolds and Related Topics (Proc. Univ. of Georgia Inst., 1961), Prentice-Hall, Englewood Cliffs, N. J., 1962, pp. 29-33. MR 25 \#4504.

2. C. E. Burgess and J. W. Cannon, Embeddings of surfaces in $E^{3}$, Rocky Mountain J. Math. 1 (1971), 259-344. MR 43 \#4008.

3. J. W. Cannon, ULC properties in neighborhoods of embedded surfaces and curves in $E^{3}$, Canad. J. Math. 25 (1973), 31-73. MR 47 \#2589.

4. R. H. Fox and E. Artin, Some wild cells and spheres in three-dimensional space, Ann. of Math (2) 49 (1948), 979-990. MR 10, 317.

5. D. S. Gillman, Side approximation, missing an arc, Amer. J. Math. 85 (1963), 459-476. MR 28 \#3407.

6. L. D. Loveland, Piercing points of crumpled cubes, Trans. Amer. Math. Soc. 143 (1969), 145-152. MR 40 \#883.

7. D. R. McMillan, Some topological properties of piercing points, Pacific J. Math. 22 (1967), 313-322. MR 35 \#7319.

8. - Piercing a disk along a cellular set, Proc. Amer. Math. Soc. 19 (1968), 153-157. MR $36 \# 3332$.

9. Harvey Rosen, Piercing points on a special arc, Proc. Amer. Math. Soc. 46 (1974), 438-442.

10. R. L. Wilder, Topology of manifolds, Amer. Math. Soc. Colloq. Publ., vol. 32, Amer. Math. Soc., Providence, R. I., 1963. MR 32 \#440.

DEPARTMENT OF MATHEMATICS, UTAH STATE UNIVERSITY, LOGAN, UTAH 84322 (Current address)

DEP ARTMENT OF MATHEMATICS, UNIVERISTY OF TENNESSEE, KNOXVILLE, TENNESSEE 37916 